\title{
Zur Kenntniss der stickstoffhaltigen Bestandtheile der Kürbiskeimlinge;
}

von

\section{E. Schulze.}

Für die Erforschung der Umwandlungen, welche die stickstoff haltigen Pflanzenbestandtheile im pflanzlichen Stoffwechsel erleiden, bilden bekanntlich die Keimlinge sehr geeignete Objekte. In den ungekeimten Samen finden sich als stickstoffhaltige Reservestoffe Eiweisssubstanzen in grosser Quantität vor, während Stickstoffverbindungen anderer Art daneben nur in verhältnissmässig geringen Mengen vorhanden sind; Keimpfianzen, welche sich eine Zeit lang unter Lichtabschluss entwickelt haben, enthalten dagegen in beträchtlicher Quantität Produkte der regressiven Stoftmetamorphose (A mide u. s. w.) - Substanzen, welche aus den stickstoffhaltigen Bestandtheilen, insbesondere aus den Eiweissstoffen der Samen sich während der Keimung gebildet haben. Man darf vermuthen, dass Umwandlungen, wie sie in keimenden Samen vorgehen, auch in den späteren Vegetationsstadien der Pflanzen die stickstoffhaltigen Pflanzenbestandtheile betreffen; für die Erforschung dieser Umwandlungen liegen aber bei den im Dunkeln gezogenen Keimpflanzen die Verhältnisse deshalb besonders günstig, weil in diesen die Unwandlungsprodukte in relativ grosser Menge sich vorinden (insbesondere in den Keimlingen stickstoffreicher Samen), weil ferner hier nicht wie in den grünen Pflanzentheilen durch das Dazwischentreten des Assimilationsprocesses eine verwickeltere Sachlage geschaffen wird.

Für die von meinen Mitarbeitern und mir in dieser Richtung ausgeführten Untersuchungen haben neben den Keimlingen der gelben Lupine hauptsächlich die Kürbiskeimlinge als Objekt gedient. Die mit letzteren erhaltenen Ergebnisse sind in einer Abhandlung zusammengestellt worden, welche ich in Verbindung mit J. Barbieri in 


\section{Schulze: Zur Kenntniss der stickstoffhaltigen}

dieser Zeitschrift ${ }^{3}$ ) früher veröffentlicht habe. Diese Arbeit zeigt manche Lücken und manche Unvollkommenheiten was leicht erklärlich ist, wenn man bedenkt, dass sie einen der ersten Versuche bildet, welche zur Erforschung der mit dem Keimungsvorgang verbundenen Umsetzung der stickstoffhaltigen Samenbestandtheile gemacht wurden. Dies veranlasste mich, die Untersuchung zu wiederholen. Ich wurde dabei aufs Beste unterstützt von den Herren Dr. E. Bosshard und E. Steiger, insbesondere haben dieselben die erforderlichen quantitativen Bestimmungen fast ohne Ausnahme ausgeführt.

Ehe ich die nun gewonnenen Resultate mittheile, will ich die früher erhaltenen kurz recapituliren. In der von J. Barbieri und mir publicirten Arbeit wurde nachgewiesen, dass man aus dem Saft der Kürbiskeimlinge Glutaminsäure abscheiden kann, nachdem man denselben zuvor mit einer Mineralsäure erhitzt hat. Da bei letzterem Vorgange auch ein Ammoniaksalz entsteht, so musste es für höchst wahrscheinlich erklärt werden, dass in den genannten Keimlingen ein Homologes des Asparagins, das Glutamin enthalten sei; doch konnte diese Annahme nicht sicher bewiesen werden, weil es nicht gelang, ein solches Amid aus dem Saft zur Abscheidung zu bringen. Neben Glutaminsäure erhielten wir auch ein wenig Asparaginsäure, deren Entstehung wir auf einen Gehalt der Keimlinge an Asparagin zurückführten; die letztere Annahme wurde dadurch fast zur Gewissheit erhoben, dass wir wenigstens in einem Falle Asparagin aus den Keimlingen zur Abscheidung bringen konnten. Ferner vermochten wir aús den Kürbiskeimlingen Tyrosin und einen im Verhalten mit Leucin übereinstimmenden Stoff zu gewinnen. Endlich konnten wir es sehr wahrscheinlich machen, dass bei der Zersetzung der Eiweisssubstanz der Kürbissamen ausserhalb des Organis. mus durch Salzsäure die einzelnen Amidosäuren in einem ganz anderen MengenverhäItniss entstehen, als bei der Spaltung jener Eiweisssubstanz in den Keimlingen.

1) Dies. Journ. [2] 20, 385-418. Kurze Mittheilungen über die Ergebnisse sind in den Ber. Berl. chem. Ges.11, 710 u. 1283 gemacht worden. 
Oben ist gesagt worden, dass der früheren Arbeit einige Mängel anhaften; es wird zweckmässig sein, dieselben näher zu bezeichnen. Erstens ist der Nachweis für das Vorbandensein von Glutamin in den Keimlingen in der früheren Arbeit nicht mit Sicherheit geführt worden; ferner haben wir das Leucin nicht in ganz reinem Zustande erhalten und konnten dasselbe daher auch nicht ganz sicher identificiren; endlich sind die quantitativen Bestimmungen, durch welche wir uns über den Gehalt der Keimlinge an Glutamin und an Ammoniak Aufschluss zu verschaffen suchten (Bestimmungen, für deren exacte Ausführung freilich auch jetzt noch die Mittel mangeln), mit Fehlern behaftet; diese Fehler entsprangen zum grossen Theil dem Umstande, dass wir über das Verhalten des damals noch nicht isolirten Glutamins gegen die bei jenen Bestimmungen zur Anwendung kommenden Reagentien keine genügende Kenntniss besassen. ${ }^{1}$ )

Durch die späteren Untersuchungen, deren Ergebnisse ich im Folgenden mittheile, ist der Nachweis für das Vorhandensein von Glutamin, Asparagin, Leucin und Tyrosin in den Kürbiskeimlingen weit vollständiger geführt worden als früher. Wir haben daneben noch einige andere stickstoffhaltige Stoffe in den genannten Keimlingen auffinden und die Quantitätsbestimmungen auf eine bessere Grundlage bringen können.

Die Kürbissamen, welche wir für unsere Versuche verwendeten, waren aus der Handlung von Metz \& Comp. in Steglitz bei Berlin bezogen und gehörten zwei verschiedenen, vom Lieferanten als „Feldkürbis“ und ,grosser gelber Speisekürbis" bezeichneten Sorten an; vorzugsweise kam die erstere Sorte zur Verwendung. Bestimmungen des Stickstoff- und des Fettgehaltes in den entschälten Samen dieser Sorte gaben folgende Zahlen:

Gesammtstickstoff $5,93 \%$ der Samentrockensubstanz Fett $52,00, "$,

1) Wir glaubten annehmen zu dürfen, dass das Verhalten des Glutamins demjenigen des Asparagins völlig analog sei, was aber nach unseren jetzigen Kenntnissen als nicht ganz zutreffend zu bezeichnen ist. 


\section{Schulze: Zur Kenntniss der stickstoffhaltigen}

Bei der Analyse der für unsere früheren Versuche verwendeten Samensorten waren folgende Zahlen gefunden worden $\left.{ }^{1}\right)$ :

$\begin{array}{lcc} & \text { Sorte A. } & \text { Sorte B. } \\ \text { Gesammtstickstoff } & 5,96 \% & 5,73 \% \\ \text { Fett (Aetherextract) } & 52,00 \% & 58,60 \%, \% \text { der Samentrockensubstanz }\end{array}$

Demnach scheint die Zusammensetzung der entschälten Kürbissamen eine ziemlich constante zu sein. Dass „nicht eiweissartige" lösliche Stickstoffverbindungen in den ungekeimten Samen nur in sehr geringer Menge vorhanden sind, ist in unserer früheren Arbeit nachgewiesen worden.

Die Keimlinge wurden in grossen mit Flusssand gefüllten Kästen aus Zinkblech in einem verdunkelten Zimmer gezogen (einige Vegetationen in irdenen, gleichfalls mit Sand gefüllten Töpfen); sie wurden mit destillirtem Wasser begossen.

Der Uebersichtlichkeit halber widme ich in Folgendem jedem der in den Keimlingen von uns aufgefundenen stickstoff haltigen Bestandtheile einen mit besonderer Ueberschrift versehenen Abschnitt.

\section{Glutamin.}

Nachdem wir gefunden hatten, dass aus dem Rübensaft das Glutamin durch Ausfällung mittelst salpetersaurem Quecksilberoxyds gewonnen werden kann, versuchten wir, es nach dem gleichen Verfahren aus Kürbiskeimlingen darzustellen. Die frischen Keimlinge wurden unter Zusatz von Sand zerrieben, der Saft durch Auspressen und darauf folgendes Auswaschen mit Wasser gewonnen. Die so erhaltene Flüssigkeit versetzten wir mit Bleiessig in schwachem Ueberschuss, entfernten die dadurch hervorgebrachte Fällung durch Filtration und fügten zum Filtrat eine Lösung von salpetersaurem Quecksilberoxyd. ${ }^{2}$ ) Es entstand ein starker weisser

1) Dies. Journ. [2] 20, 392.

2) Zur Darstellung dieser Lösung wurde ein krystallinisches Präparat von salpetersaurem Quecksilberoxyd mit kaltem Wasser behandelt und das ungelöst bleibende basisehe Salz durch Filtration entfernt. 
Niederschlag, welcher abfiltrirt und mit kaltem Wasser gewaschen, sodann in Wasser aufgerührt und durch Schwefelwasserstoff zersetzt wurde. Die rom Schwefelquecksilber abfiltrirte Flüssigkeit neutralisirten wir mit Ammoniak und dunsteten sie im Wasserbade bei gelinder Wärme auf ein geringes Volumen ein ${ }^{1}$; sie wurde dann unter eine Glasglocke über Schwefelsäure gestellt und der weiteren langsamen Verdunstung überlassen.

In den ersten Versuchen lieferten diese Flüssigkeiten gar keine Krystallisationen von Glutamin; wir erhielten aus denselben aber einen im Verhalten mit Glutaminsäure übereinstimmenden Körper, als wir sie nach demjenigen Verfahren behandelten, welches uns früher zur Darstellung der genannten Amidosäure aus dem Saft der Kürbiskeimlinge gedient hat. ${ }^{2}$ Aus diesem Ergebniss mussten wir schliessen, dass in der bei Zerlegung des Quecksilberniederschlages erhaltene Flüssigkeit Glutamin vorhanden war, aber durch Beimengungen am Auskrystallisiren verhindert wurde. Wir unterwarfen daher in einem zweiten Versuche den aus den Kürbiskeimlingen gewonnenen Saft einer fraktionirten Ausfällung mit salpetersaurem Quecksilberoxyd. Der zuletzt erhaltene Theil des Niederschlags lieferte nun, als er in der oben beschriebenen Weise verarbeitet wurde, in geringer Menge Krystalle, welche im Aussehen und im Verhalten mit dem aus Rüben gewonnenen Glutamin übereinstimmten, gemengt mit etwas Tyrosin, von welchem sie jedoch durch Lösen in schwach erwärmtem Wasser leicht getrennt werden konnten. ${ }^{3}$ ) Die Quantität, in welcher wir diese Krystalle erhielten, war jedoch nicht gross genug, um durch eingehende Untersuchung ihre Identität mit Glutamin feststellen $z u$ können.

1) Da die Flüssigkeit während des Eindampfens allmählich wieder saure Reaction annimmt, so wurden von Zeit zu Zeit einige Tropfen einer Lösung von kohlensaurem Ammoniak zugefügt. S. 393.

2) Man vergl. in Betreff dieses Verfahrens die frühere Arbeit,

3) Die Ergebnisse dieser Versuche sind schon in einer Publication über das Glutamin (Ber. Berl. chem. Ges. 16, 312) erwähnt worden. 
Zu einem besseren Resultate führten Versuche, welche wir im verflossenen Winter ausgeführt haben. $\mathrm{Da}$ in den Lupinenkeimlingen besonders die Axenorgane (Wurzel und hypocotyles Glied etc.) sehr asparaginreich sind, so war zu vermuthen, dass in den Kürbiskeimlingen diese Theile viel Glutamin enthalten. Wir benutzten daher für neue Versuche nur die Axenorgane dieser Keimlinge. Dieselben wurden in frischem Zustande zerrieben, der Saft ausgepresst und in der oben beschriebenen Weise behandelt. Bei Verarbeitung der so gewonnenen Quecksilberniederschläge erhielten wir Flüssigkeiten, welche nach dem Eindunsten auf ein geringes Volumen ziemlich reichliche Krystallisationen von Glutamin lieferten. ${ }^{1}$ ) Aus sechs Vegetationen von Kürbiskeimlingen, welche aus zwei verschiedenen Samensorten dargestellt waren, erhielten wir in solcher Weise Glutamin. Dasselbe glich völlig dem aus dem Rübensaft dargestellten; es krystallisirte wie dieses in feinen weissen Nadeln, welche kein Krystallwasser enthielten, sich ziemlich schwer in kaltem, leicht in heissem Wasser lösten, und durch Alkalien rasch unter Ammoniakbildung zersetzt wurden. Aus einer in der Hitze mit Kupferoxydhydrat gesättigten wässerigen Lösung der Krystalle schied sich beim Erkalten eine dem Glutaminkupfer gleichende blaue krystallinische Verbindung aus. Wir bestimmten in derselben den Kupfergehalt und erhielten dabei folgende Resultate:

1) 0,2019 Grm. Substanz (bei $100^{\circ}$ getrocknet) gaben, im Saucrstoffistrome verbrannt, $0,0452 \mathrm{Grm}$. $\mathrm{CuO}$.

2) 0,2094 Grm. Substanz (von einem zweiten Präparat) gaben 0,0464 Grm. CuO.

\begin{tabular}{cccc}
\multicolumn{2}{c}{ Berechnet } & \multicolumn{2}{c}{ Gefunden. } \\
für $\left(\mathrm{C}_{\tilde{5}} \mathrm{H}_{3} \mathrm{~N}_{2} \mathrm{O}_{3}\right)_{2} \mathrm{Cu}$. & \multicolumn{1}{c}{1.} & 2. \\
$\mathrm{Cu}$ & $17,94 \%$ & $17,86 \%$ & $17,69 \%$
\end{tabular}

Dass die beschriebene Substanz Glutamin war, wird ausser durch die im Vorigen mitgetheilten Thatsachen noch dadurch bewiesen, dass sie bei der Zersetzung Glutamin-

1) Wenigstens dann, wenn die Keimlinge drei Wochen oder länger vegetirt hatten. Jüngere Keimlinge lieferten weniger Glutamin. 
säure lieferte. Die Darstellung der letzteren geschah in folgender Weise: Wir kochten das Glutamin mit Barytwasser, bis kein Ammoniakgeruch mehr zu bemerken war, fällten den Baryt sodann durch Schwefelsäure aus, filtrirten und verdunsteten das Filtrat zur Krystallisation. Die so erhaltenen Krystalle wurden in Wasser gelöst, die Lösung mit Kupferoxydhydrat gesättigt, das beim Eindunsten der Flüssigkeit sich ausscheidende Kupfersalz wieder durch Schwefelwasserstoff zerlegt. Die so dargestellte Amidosäure stimmte im Aussehen und im Verhalten mit Glutaminsäure überein. Die Salzsäureverbindung derselben krystallisirte in glänzenden Tafeln. Hr. Professor K. Haushofer in München hatte auf meine Bitte die Güte, diese Verbindung krystallographisch zu untersuchen; er theilte mir über die Resultate dieser Untersuchung Folgendes mit:

„Die Beschaffenheit der übersendeten Krystalle gestattete eine hinreichend genaue Untersuchung derselben. Aus den Winkelmessungen sowie aus den optischen Verhältnissen ergibt sich die vollständige Identität derselben mit der von Becke ${ }^{1}$ ) untersuchten salzsauren Glutaminsäure."

Diese Thatsachen machen es zweifellos, dass die Kürbiskeimlinge Glutamin enthalten.

\section{A sparagin.}

Dass die Kürbiskeimlinge zuweilen Asparagin in ziemlich grosser Quantität enthalten, zeigte sich in den behufs der Abscheidung des Glutamins unternommenen Versuchen; in einem Falle nämlich lieferte die Flüssigkeit, welche bei Verarbeitung des durch salpetersaures Quecksilberoxyd im Saft hervorgebrachten Niederschlags erhalten wurde, eine der Menge nach beträchtliche Krystallisation von Asparagin. Zur Identificirung desselben diente, neben dem charakteristischen Aussehen und dem Verhalten der Krystalle ${ }^{2}$, eine Bestimmung des Krystallwassergehalts:

1) Zeitschr. f. Krystallographie 5, 366.

2) Das Asparagin ist auch ohne Ausführung analytischer Bestimmungen und ohne Krystallmessungen an folgenden Merkmalen zu er- 


\section{Schulze: Zur Kenntniss der stickstoffhaltigen}

$0,309 \mathrm{Grm}$. Substanz verloren bei $100^{\circ} 0,0370 \mathrm{Grm} .=11,97^{\circ} \%$ an Gewicht (während die Theorie 12,0\% verlangt).

Dass in diesem Falle das Glutamin in den Keimlingen durch Asparagin vertreten war, darf wohl als sehr wahrscheinlich bezeichnet werden. ${ }^{1}$ ) Es gelang uns aber auch nachzuweisen, dass in denjenigen Kürbiskeimlingen, welche Glutamin enthalten, neben dem letzteren A sparagin in geringer Menge sich vorfindet, und zwar kommt dasselbe in den Cotyledonen der Keimlinge vor. Der Nachweis geschah in folgender Weise: Die getrockneten Cotyledonen wurden zerrieben und sodann mit heissem Wasser extrahirt, die Extracte zuerst mit Bleiessig in schwachem Ueberschuss, dann (nach der Filtration) mit einer Lösung von salpetersaurem Quecksilberoxyd versetzt; der durch letzteres Reagens hervorgebrachte starke Niederschlag wurde in Wasser aufgerührt und durch Schwefelwasserstoff zersetzt, die vom Schwefelquecksilber abfiltrirte Flüssigkeit ebenso behandelt, wie es früher beschrieben worden ist. Aus dieser Flüssigkeit schied sich zuerst eine unkrystallinische Substanz aus (einen später noch zu beschreibenden Stoff einschliessend), welcher jedoch einige Asparaginkrystalle beigemengt waren. Die von dieser Ausscheidung abfiltrirte Mutterlauge lieferte bei weiterem Verdunsten noch mehr Asparagin. Die vorher erwähnte

kennen: Es krystallisirt in durchsichtigen Krystallen, welche bei langsamer Ausbildung eine bedeutende Grösse erreichen und bei $100^{\circ}$ ihr Krystallwasser verlieren. Sättigt man die wässrige Lösung in der Hjtze mit Kupferoxydhydrat, so erhält man eine lasurblaue Flüssigkeit; beim Erkalten scheidet sich aus derselben eine fein krystallinische Kupferverbindung aus, welche (wenigstens in reinem Zustande) schön blau mit einem Stich in's Violette ist. Beim Erwärmen mit verdünnter Kalilaüge entwickelt das Asparagin lebhaft Ammoniak; erhitzt man es mit verdünnter Salzsäure, so entsteht in der Flüssigkeit ein Ammoniaksalz, Diese Reactionen giebt freilich auch das Glutamin; da dasselbe aber in feinen Nadeln ohne Krystallwasser krystallisirt, so ist eine Verwechslung kaum möglich. Will man bei der Identificirung des Asparagins eine analytische Bestimmung zu Hülfe nehmen, so empfiehlt sich für diesen Zweck die leicht ausführbare Krystallwasserbestimmung.

1) Eine Beobachtung, welche $z u$ dem gleichen Schlusse führt, ist schon in der früheren Arbeit mitgetheilt worden. 
amorphe Substanz liess sich durch Abschlemmen vom beigemengten Asparagin trennen. Letzteres wurde dann durch Umkrystallisiren gereinigt. Bei einer späteren Darstellung erhielten wir neben dem Asparagin auch Tyrosin; die beiden Körper lassen sich, da ihre Löslichkeit im Wasser eine so verschiedene ist, ohne Schwierigkeit von einander trennen.

Das so gewonnene Präparat stimmte im Aussehen und Verhalten vollkommen mit Asparagin überein. Bestimmungen des Krysallwasser- und des Stickstoffgehalts gaben folgende Resultate:

1) $0,569 \mathrm{Grm}$. Subst. verloren bei $100^{\circ} 0,0675 \mathrm{Grm}$. an Gewicht.

2) $0,4095, " \quad " 100^{\circ} 0,0490 "$ " " "

3) $0,319 "$ " gaben nach der Methode von Kjeldahl $0,05857 \mathrm{Grm} . \mathrm{N}$ in Ammoniakform (= 15,15 Ccm. Barytwasser). $\left.{ }^{1}\right)$

Berechnet

für $\mathrm{C}_{4} \mathrm{H}_{8} \mathrm{~N}_{2} \mathrm{O}_{3}+\mathrm{H}_{2} \mathrm{O}$.

$$
\mathrm{H}_{2} \mathrm{O} \quad 12,00 \%
$$

Gefunden.

\begin{tabular}{ccc}
\hline 1. & 2. & 3. \\
$12,05 \%$ & $11,96 \%$ & - \\
- & - & $18,36 \%$
\end{tabular}

Aus allen von uns in der beschriebenen Weise untersuchten Cotyledonen (von mehreren Vegetationen von Kürbiskeimlingen stammend) vermochten wir Asparagin abzuscheiden. Diese Thatsache steht im Einklang mit dem in der früheren Arbeit erbrachten Nachweis, dass man aus den mit Salzsäure gekochten Extracten aus Kürbiskeimlingen neben Glutaminsäure auch etwas Asparaginsäure gewinnen kann.

Aus den glutaminhaltigen Niederschlägen, welche in den aus den Axenorganen der Keimlinge dargestellten Extracten durch salpetersaures Quecksilberoxyd hervorgebracht wurden, haben wir in keinem Falle Asparagin erhalten.

\section{Tyrosin.}

Die Abscheidung des Tyrosins aus den Kürbiskeimlingen wurde von J. Barbieri und $\mathrm{mir}^{2}$ ) in folgender Weise aus-

1) Titre des Barytwassers: $1 \mathrm{Ccm} .=0,003866 \mathrm{Grm} . \mathrm{N}$.

2) Man vergl. die frühere Abhandlung S. 401. 
geführt: Der aus den zerriebenen frischen Keimlingen durch Auspressen und nachfolgendes Auswaschen mit Wasser gewonnene Saft wurde durch Erhitzen vom Eiweiss befreit, dann auf ein geringes Volumen eingedunstet und mit viel Weingeist versetzt; die dadurch hervorgebrachte Fällung entfernten wir durch Filtration und dunsteten das Filtrat zum dünnen Syrup ein. Aus letzterem schied sich nach einiger Zeit Tyrosin in kleinen warzenförmigen Krystallgruppen ans. Aus vier nach einander untersuchten Vegetationen von Kürbiskeimlingen vermochten wir in solcher Weise Tyrosin zur Abscheidung zu bringen. Bei der späteren Untersuchung gingen wir nicht speciell auf den Nachweis des Tyrosins aus, erhielten dasselbe aber in vielen Fällen als Nebenprodukt, so z. B. in den Versuchen, welche behufs der Abscheidung des Glutamins aus den Keimlingen unternommen wurden. Die durch salpetersaures Quecksilberoxyd in den Extracten hervorgebrachten Niederschläge, aus denen wir das Glutamin zu gewinnen suchten, enthielten in den meisten Fällen etwas Tyrosin. Aus den bei Zerlegung dieser Niederschläge erhaltenen Flüssigkeiten krystallisirt dasselbe mit dem Glutamin aus; die Trennung beider Körper liess sich natürlich leicht bewerkstelligen, da dieselben in Bezug auf die Löslichkeit im Wasser sehr von einander abweichen.

Auch in den Cotyledonen der Kürbiskeimlinge liess sich auf diesem Wege Tyrosin nachweisen. Als die aus den getrockneten Cotyledonen dargestellten wässrigen Extracte zuerst mit Bleiessig, dann mit salpetersaurem Quecksilberoxyd versetzt, und die durch letzteres Reagens hervorgebrachten Niederschläge in der früher beschriebenen Weise verarbeitet wurden, resultirten Flüssigkeiten, aus denen beim Verdunsten Tyrosin neben anderen Körpern (Asparagin etc.) sich ausschied; wegen seiner Schwerlöslichkeit konnte es leicht von den Beimengungen getrennt werden. Es wurde jedoch in solcher Weise nur wenig Tyrosin erhalten, und in einigen Fällen fehlte dasselbe ganz.

$\mathrm{Da}$ in einer mit salpetersaurem Quecksilberoxyd versetzten Tyrosinlösung nur nach und nach eine Fällung ent- 
steht $^{1}$ ) so liegt auf der Hand, dass in die durch das genannte Reagens in den Extracten hervorgebrachten Niederschläge mehr oder weniger Tyrosin eingehen wird, je nachdem man dieselben nach kürzerer oder längerer Zeit aufs Filter bringt. Wemn man die aus den Cotyledonen und den Axenorganen der Keimlinge dargestellten wässrigen Extracte mit salpetersaurem Quecksilberoxyd in schwachem Ueberschuss versetzt, dann sofort filtrirt und zum Filtrat noch etwas von dem genannten Reagens zusetzt, so scheidet sich nach und nach ein ziemlich dichter Niederschlag aus, welcher bei der Zerlegung mittelst Schwefelwasserstoff eine nicht unbeträchtliche Tyrosinmenge liefert.

Enḋlich haben wir Tyrosin auch bei der im folgenden Abschnitt zu beschreibenden Abscheidung von Leucin aus den Keimlingen als Nebenprodukt erhalten. Aus allen diesen Beobachtungen darf wohl geschlossen werden, dass Tyrosin ein constanter Bestandtheil der Kürbiskeimlinge ist.

Nach dem von Hlasiwetz und Habermann ${ }^{2}$ ) angegebenen Verfahren gereinigt, bildete das aus den Keimlingen abgeschiedene Tyrosin feine, glänzende, weisse Nadeln, welche sowohl im Aussehen (auch unter dem Mikroskop) wie im Verhalten mit dem aus Eiweissstoffen oder aus Horn dargestellten Tyrosin vollständig übereinstimmen. ${ }^{3}$ )

Als eine Lösung unseres Präparats in kochendem Wasser mit Kupferoxydhydrat gesättigt und dann der Ruhe überlassen wurde, schieden sich aus derselben kleine dunkelblaue Prismen aus, welche im Aussehen (auch unter dem Mikroskop) mit Tyrosinkupfer vollkommen übereinstimmten. Eine Kupferbestimmung in diesem Salz gab folgende Resultate:

1) Wenigstens dann, wenn die Lösung des salpetersauren Quecksilberoxyds sauer ist.

2) Dies. Journ. [2] 7, 397.

3) Da ein in Ratanhin-Extract aufgefundeüer Körper, das Ratan hin (welches wahrscheinlich Methyl-Tyrosin ist) die Hoffmann'sche und die Piria'sche Tyrosin-Reaction giebt, so ist es vielleicht nicht überflüssig, auf eine Verschiedenheit hinzuweisen, welche dasselbe von dem von uns aus Kürbiskeimlingen dargestellten Präparat und vom 


\section{Schulze: Zur Kenntniss der stickstoffbaltigen}

$0,1715 \mathrm{Grm}$. Substanz, zuerst über Schwefelsäure, dann im Luftbade getrocknet ${ }^{1}$ ), gaben beim Glühen (unter Zuleiten von Sauerstoff) 0,0325 Grm. Cao.

$$
\begin{array}{cc}
\text { Berechnet } & \text { Gefunden. } \\
\text { für } \left.\mathrm{C}_{9} \mathrm{H}_{10} \mathrm{NO}_{3}\right)_{2} \mathrm{Cu} . & 15,13 \% \\
\mathrm{Cu} \quad 14,95 \% & \\
\multicolumn{2}{c}{\text { Leucin. }}
\end{array}
$$

Der von J. Barbieri und mir für Leucin erklärte Bestandtheil der Kürbiskeimlinge hatte sich, wie in der früheren Abhandlung erwähnt worden ist, aus der vom Tyrosin abfiltrirten Mutterlauge beim weiteren Verdunsten derselben abgeschieden; nach dem Umkrystallisiren aus ammoniakhaltigem Weingeist zeigte er Aussehen und Eigenschaften des nicht ganz reinen Leucins. Er wurde nur in sehr geringer Menge erhalten, und seine Abscheidung aus dem betreffenden Extract gelang nur mit Mühe.

Bei den späteren Versuchen schlugen wir zur Gewinnung dieses Körpers einen ganz anderen Weg ein. Bei der Untersuchung der Lupinenkeimlinge hatten wir gefunden, dass die in denselben vorhandenen Amidosäuren vorzugsweise in den Axenorganen enthalten sind und sich am leichtesten aus den weingeistigen Extracten gewinnen lassen; diese Erfahrungen suchten wir für die Untersuchung der Kürbiskeimlinge zu verwerthen. Die nach Entfernung der Cotyledonen übrig bleibenden Theile dieser Keimlinge wurden getrocknet, zerrieben und sodann in der Wärme mit Weingeist von 91-92 Vol. Proc. extrahirt. Nachdem aus den Extracten der Weingeist abdestillirt worden war, nahmen

Tyrosin anderer Herkunft zeigt. Wenn man Ratanhin mit etwas mässig starker Salpetersäure erwärmt, so erhält man eine rosenrothe Flïssigkeit, welche später violett und blau wird; diese charakteristische Reaction soll sehr empfindlich sein (nach Kreitmayer, man vergl. Beilstein, Handb. d. organ. Chem. S. 1967). Das aus Kürbiskeimlingen abgeschiedene Tyrosin gab, ebenso wie das aus Eiweissstoffen gewonnene, bei gleicher Behandlung nur eine gelbe Flüssigkeit.

1) Beim Trocknen im Luftbade verlor das Salz nichts an Gewicht. Die von uns für das Salz gefundene Zusammensetzung entspricht den von Hofmeister, sowie von Erlenmeyer und Lipp gemachten Angaben. 
wir den Rückstand mit Wasser auf und versetzten die Flüssigkeit mit Bleiessig in schwachem Ueberschuss. Den so erzeugten Niederschlag entfernten wir durch Filtration, bęfreiten das Filtrat mittelst Schwefelwasserstoff vom Blei und dunsteten es sodann im Wasserbade bis zum dünnen Syrup ein. Auf letzterem bildete sich beim Erkalten eine Haut, beim Stehen schied sich aus der stark braunen Flüssigkeit nach und nach eine dem unbewaffneten Auge ganz amorph erscheinende Substanz in nicht unbeträchtlicher Menge aus. Mit Hülfe eines Zeugfilters liess sie sich von der Mutterlauge trennen. Nachdem sie zuerst mit verdünntem, dann mit stärkerem Weingeist gewaschen, zwischen Fliesspapier stark abgepresst und getrocknet worden war, bildete sie eine gelblich oder bräunlich gefärbte, zerreibbare Masse. Sie wurde nun zerrieben und in der Wärme mit starkem Weingeist unter Zusatz von etwas concentrirter Ammoniakflussigkeit behandelt. Der grösste Theil ging in Lösung, zurück blieb etwas Tyrosin. Die Lösung wurde in einem Becherglas über Schwefelsäure gestellt. Schon während des Erkaltens schied sich eine im Aussehen dem nicht ganz reinen Leucin gleichende weisse Substanz aus, deren Menge sich beim Verdunsten des Ammoniaks noch vermehrte. Nach nochmaligem Umkrystallisiren zuerst aus ammoniakhaltigem, dann aus mit Wasser verdünntem Weingeist ${ }^{1}$ ) bildete sie glänzende Blättchen, welche im Aussehen und im Verhalten mit reinem Leucin übereinstimmten. Beim Erhitzen im Proberöhrchen verflüchtigte sie sich vollständig zu einem weissen wolligen Sublimat. Als die heisse wässrige Lösung mit Kupferoxyd gesättigt wurde, schied sich schon in der Wärme eine schuppig krystallinische, dem Leucin-Kupfer gleichende Verbindung aus; die gleiche Ausscheidung erfolgte aus jener Lösung auf Zusatz von Kupferacetat-Solution. Durch diese Reaction unterscheidet sich das Leucin von der in den Lupinenkeimlingen von uns aufgefundenen Amidovaleriansäure ${ }^{2}$ ), welche im Uebrigen dem Leucin sehr

1) Eine geringe Tyrosin-Menge, durch welche das Leucin anfangs noch verunreinigt war, blieb dabei zurück.

2) Man vergl. dies. Journ. [2] 27, 353 . 


\section{Schulze: Zur Kenntniss der stickstoffhaltigen}

ähnlich ist. Einen weiteren Beweis dafür, dass Leucin vorlag, lieferten die nach Kjeldahl's Methode ${ }^{1}$ ) in zwei verschiedenen Krystallisationen ausgeführten Stickstoffbestimmungen:

1) 0,2455 Grm. Substanz (bei $100^{\circ}$ getrocknet) gaben 0,026289 Grm. N ( $=6,80 \mathrm{Ccm}$. Barytwasser). $\left.{ }^{2}\right)$

2) 0,2070 Grm. Substanz (bei $100^{\circ}$ getrocknet) gaben 0,021843 Grm. N (=5,65 Cem. Barytwasser).

\begin{tabular}{cccc}
\multicolumn{2}{c}{ Berechnet } & \multicolumn{2}{c}{ Gefunden. } \\
für $\mathrm{C}_{6} \mathrm{H}_{13} \mathrm{NO}_{2}$. & \multicolumn{1}{c}{1.} & 2. \\
$\mathrm{~N}$ & $10,69 \%$ & $10,71 \%$ & $10,55 \%$
\end{tabular}

Die beim Umkrystallisiren des Leucins übrig gebliebene Mutterlauge wurde zur Trockne verdunstet, der Rückstand mit verdünnter Schwefelsäure und Kaliumbichromat einige Stunden am Rückflusskühler erhitzt, dabei trat der Geruch des Benzaldehyds auf (freilich nicht ganz rein); beim Erkalten schieden sich aus der Flüssigkeit einige Krystallblättchen aus, welche beim Erhitzen im Röhrchen u. s. w. das Verhalten der Benzoësäure zeigten. Die Menge derselben war jedoch nicht gross genug, um sie in reinen $\mathrm{Zu}$ stand überführen und mit Sicherheit identificiren zu können.

Dieses Ergebniss macht es wahrscheinlich, dass dem aus den Kürbiskeimlingen gewonnenen Rohleucin eine ganz ge ringe Menge von Phenylamidopropionsäure beigemengt war. Diese in den Lupinenkeimlingen von uns aufgefundene Substanz liefert bekanntlich bei der Oxydation Benzaldehyd und Benzoësäure.

Nach dem Umkrystallisiren war das aus den Keimlingen abgeschiedene Leucin frei von Phenylamidopropionsäure, wie u. a. auch aus seinem Verhaiten im Proberöhrchen geschlossen werden kann. Während sich Leucin bekanntlich beim Erhitzen im Röhrchen ohne zu schmelzen verflüchtigt, schmilzt die Phenylamidopropionsäure bei gleicher Behandlung unter Bildung charakteristischer Zersetzungsprodukte,

1) Dass diese Methode für die Analyse von Amidosäuren sehr brauchbar ist, ergiebt sich aus den von E. Bosshard (Zeitschr. für analyt. Chem. 24, 299) gemachten Versuchen.

2) Titre des Barytwassers: 1 Ccm. $=0,003866$ Grm. N. 
während ein geringer Theil unzersetzt sublimirt ${ }^{1}$ ); es ist daher leicht, beide Körper durch ihr Verhalten im Röhrchen zu unterscheiden und in einem Leucinpräparat eine Beimengung von Phenylamidopropionsäure zu erkennen.

\section{Vernin.}

Aus den Flüssigkeiten, welche bei Verarbeitung der in den wässrigen Cotyledonen-Extracten durch salpetersaures Quecksilberoxyd hervorgebrachten Niederschläge in der oben (beim Asparagin) beschriebenen Weise erhalten wurden, schied sich neben Asparagin ein amorpher Körper aus, welcher nach dem Wiederauflösen in wenig heissem Wasser krystallinisch wurde. Von den beigemengten Asparaginkrystallen liess er sich durch Abschlemmen und darauf folgendes Umkrystallisiren aus heissem Wasser trennen. Seine Quantität war nicht ganz unbeträchtlich. Die Cotyledonen anderer etwas älterer Keimlinge lieferten ihn nur in höchst geringer Menge, auch war in letzterem Falle ziemlich viel Tyrosin beigemengt, welches die Isolirung der vorhin erwähnten Substanz erschwerte.

Nach der Reinigung krystallisirte dieser Körper aus Wasser in sehr dünnen seideglänzenden Prismen, welche sich schwer in kaltem, leicht in kochendem Wasser lösten. Die wässrige Lösung, welche neutral reagirte, wurde nicht gefällt durch Bleizucker und Kupferacetat, dagegen durch Pikrinsäure, durch Phosphorwolframsäure (unter Zusatz von Salzsäure) und durch Silbernitrat. Die weitere Untersuchung zeigte, dass dieser Körper in seinen Eigenschaften vollkommen mit einer stickstoffreichen Substanz übereinstimmt, welche wir zuerst in jungen Wicken- und Rothkleepflanzen aufgefunden haben ${ }^{2}$ ), und welcher wir den Namen Vernin beilegen wollen. Eine ausführliche Publication über diesen Körper soll demnächst erfolgen; wir begnügen uns an dieser

1) Nur bei sehr langsamem Erhitzen sublimirt ein grösscrer Theil der Phenylamidopropionsäure unzersetzt.

2) Eine vorläufige Mittheilung über diesen Körper ist in der Zcitschrift für physiologische Chemie 9, 444 gemacht worden. 


\section{Schulze: Zur Kenntniss der stickstoffhaltigen}

Stelle auf sein Vorhandensein in den Cotyledonen der Kürbiskeimlinge aufmerksam gemacht zu haben.

\section{Xanthinkörper.}

Die bei Verarbeitung der Quecksilberniederschläge erhaltenen Flüssigkeiten enthielten neben Glutamin, Asparagin, Tyrosin und Vernin stets Xanthinkörper. ${ }^{1}$ ) Wir fältten dieselben aus den Mutterlaugen, welche nach dem Auskrystallisiren der vorher genannten Körper übrig blieben, mit ammoniakalischer Silbernitratlösung aus. Die so erhaltenen Niederschläge lösten wir in heisser verdünnter Salpetersäure vom specif. Gewicht 1,1; in dieser Lösung schieden sich beim Erkalten feine Krystalle aus, welche mit grosser Wahrscheinlichkeit als Hypoxanthin- und Guanin-Silbernitrat anzusprechen waren. Wir krystallisirten dieselben aus verdünnter Salpetersäure um und zersetzten sie sodann unter Zusatz von etwas verdünnter Salzsäure mittelst Schwefelwasserstoff. Die vom Schwefelsilber abfiltrirte Flüssigkeit wurde mit Ammoniak übersättigt und dann eingedunstet. Während des Verdunstens schied sich eine das Verhalten des Guanins zeigende, bräunlich gefärbte Substanz aus. Sie löste sich nicht in Ammoniakflüssigkeit, dagegen in warmer, verdünnter Salzsäure; aus der stark concentrirten Lösung schied sich das salzsaure Salz in feinen Krystallnadeln aus. Die Lösung derselben gab mit Pikrinsäure die von Capranica $a^{2}$ ) beschriebene Guaninreaction.

Die vom Guanin abfiltrirte ammoniakalische Lösung gab mit Silbernitrat (unter Ammoniakzusatz) eine starke flockig-gallertartige Fällung, welche abfiltrirt, ansgewaschen und unter Zusatz von etwas Essigsäure durch Schwefelwasserstoff zerlegt wurde. Die vom Schwefelsilber abfiltrirte Lösung wurde eingedunstet, der Rückstand zur Entfernung von essigsaurem Ammoniak mit Weingeist behandelt. Es blieb eine schwach gelblich gefärbte Substanz zurück, welche

l) Es sei uns gestattet, unter dieser Bezeichnung Hypoxanthin, Xanthin, Guanin und verwandte Körper zusammenzufassen.

2) Zeitschr. f. physiolog. Chem. 4, 233. 
sich schwer in kaltem, leichter in kochendem Wasser löste; aus dieser Lösung schied sie sich in mikroskopischen Nadeln aus. Ihre wässrige Lösung gab mit ammoniakalischer Silbersolution eine gallertartige durchscheinende Fällung; sie wurde auch durch Kupferacetat (beim Kochen) gefällt.1) Aus der Auflösung der Substanz in kochender concentrirter Salzsäure schied sich beim Erkalten das salzsaure Salz in kleinen glänzenden Blättchen aus; die wässrige Lösung desselben verlor beim Eindunsten Salzsäure. Als eine Lösung der Substanz in Salpetersäure im Wasserbade zur Trockne verdunstet wurde, blieb ein farbloser Rückstand, welcher durch Natronlauge nur ganz schwach gelb gefärbt wurde.

Die im Vorigen beschriebene Substanz zeigte also im Wesentlichen das Verhalten des Hypoxanthins; doch stimmte der Silbergehalt ihrer Silbernitratverbindung nicht genau mit demjenigen des Hypoxanthin-Silbernitrats überein. Zur Darstellung jener Verbindung wurde der Niederschlag, welcher in der rom Guanin abfiltrirten Flüssigkeit (m. vgl. oben) auf Zusatz von ammoniakalischer Silberlösung entstand, in heisser verdünnter Salpetersäure vom spec. Gew. 1,1 gelöst, die beim Erkalten ausgeschiedene Substanz dann noch zweimal aus verdünnter Salpetersäure umkrystallisirt.

$0,384 \mathrm{Grm}$. des so erhaltenen, über Schwefelsäure getrockneten Salzes gaben 0,1875 Grm. Chlorsilber $=0,1411 \mathrm{Grm}$. oder $36,7 \% \mathrm{Ag}$, während die Formel des Hypoxanthinsilbernitrats $35,3 \%$ Ag verlangt.

Es ist wohl das Wahrscheinlichste, dass hier nicht ganz reines Hypoxanthin vorlag.

\section{Peptone.}

Ueber das Vorkommen von Peptonen in den Kürbiskeimlingen haben J. Barbieri und ich einige Versuche angestellt, deren Ergebnisse früher schon zur Publication gelangt sind $\left.{ }^{2}\right)$; ich reproducire dieselben hier in aller Kürze. Wir extrahirten Kürbiskeimlinge mit $W$ asser, befreiten die

1) Die Farbe des so erhaltenen Niederschlages differirte von derjenigen, welche ein durch Kupferacetat in einer Hypoxanthin-Lösung hervorgebrachter Niederschlag gewöhnlich besitzt.

2) Journ. f. Landwirthschaft, 29. Jahrg., s. 285 . 
Extracte möglichst vollständig von den Eiweisssubstanzen, säuerten sie dann mit Salzsäure oder Schwefelsäure an und versetzten sie mit Phosphorwolframsäure. Die so erhaltenen Niederschläge wurden, nach dem Abfiltriren und Auswaschen, durch Barytwasser zersetzt, die von den unlöslichen Barytverbindungen abfiltrirten Flüssigkeiten mit Natronlauge und verdünnter Kupfersulfatlösung versetzt. Es wurden stets Färbungen erhalten, welche auf das Vorhandensein von Peptonen deuteten; doch waren diese Färbungen nur sehr schwach. Wir verglichen sie mit denjenigen, welche in Fibrinpeptonlösungen von bekannter Concentration durch Kupfersulfat und Natronlauge hervorgebracht wurden. Aus diesen colorimetrischen Bestimmungen berechnete sich für die Trockensubstanz der Kürbiskeimlinge meist nur ein Peptongehalt von einigen Zehntel-Procent (nur ein Versuch gab ein etwas höheres Resultat). Auf Genauigkeit können die Ergebnisse dieser Bestimmungen freilich kaum Anspruch machen; doch berechtigen sie zu der Schlussfolgerung, dass die in den Kürbiskeimlingen enthaltene Peptonmenge nur sehr gering gewesen sein kann.

\section{Ammoniaksalze.}

Für den Nachweis derselben benutzten wir die Fällbarkeit des Ammoniaks durch Phosphorwolframsäure. Die durch Erhitzen im Wasserbade und darauf folgendes Versetzen mit Bleizucker- oder auch mit Gerbsäurelösung von den Eiweissstoffen befreiten Extracte wurden angesäuert und sodann mit Phosphorwolframsäure in starkem Ueberschuss versetzt, die so erhaltenen Niederschläge nach mehrstündigem Stehen abfiltrirt, mit säurehaltigem Wasser ${ }^{1}$ ) ausgewaschen und sodann mit Wasser und gebrannter Magnesia der Destillation unterworfen. Die so erhaltene Ammoniakmenge betrug für Keimlinge, welche $2-3$ Wochen alt waren, $0,20-0,44 \% \mathrm{NH}_{3}$, bezogen auf die Keimpflanzentrockensubstanz. Die einzelnen Zahlen theilen wir weiter unten mit.

1) Der Waschtlüssigkeit war etwas Salzsäure und etwas Phosphorwolframsäure zugesetzt. 
Salpetersaure Salze.

Eine merkwürdige Thatsache ist das Vorkommen von salpetersauren Salzen in den Kürbiskeimlingen. Die erste dahingehende Beobachtung machten wir schon im Jahre 1881, Als wir getrocknete Kürbiskeimlinge, welche bei einer relativ hohen Temperatur ${ }^{1}$ ) in Flusssand gezogen worden waren, mit Weingeist von ca. 90 Vol.-Proc. in der Wärme extrahirten, und die Extracte der Ruhe überliessen, schieden sich aus diesen Extracten Krystallnadeln aus, welche sich bei näherer Untersuchung als Kaliumnitrat erwiesen. Der Gehalt der Keimpflanzentrockensubstanz an Kaliumnitrat, berechnet nach den Ergebnissen der nach Schlö. sing's Methode ausgeführten Salpetersäurebestimmungen, betrug für zwei verschiedene Vegetationen von Keimlingen $5-6 \%$. Diesen Zahlen kommt deshalb kein grosser Werth $z u$, weil der Sand, in welchem die Keimpflanzen erwuchsen, vor der Verwendung nicht untersucht wurde und eine Verunreinigung desselben durch Nitrate, wenn auch sehr unwahrscheinlich, doch nicht ganz unmöglich ist. Auch wurden jene Keimlinge während ihres Wachsthums mit Wasser aus der Züricher Wasserleitung begossen, welches zwar sehr arm an Nitraten, aber doch sicherlich nicht ganz frei von solchen ist.

Auch Kürbiskeimlinge, welche später in ausgewaschenem Flusssand bei Zimmertemperatur gezogen und während ihres Wachsthums nur mit destillirtem Wasser begossen worden waren, enthielten Nitrate. Die nach Schlösing's Methode ausgeführten Bestimmungen gaben für zwei Vegetationen solcher Keimlinge die folgenden, auf Kaliumnitrat berechneten Resultate. ${ }^{2}$ )

1) In einem Raume, dessen ziemlich constante Temperatur nicht unter $25^{\circ}$ sank.

2) Analytische Belege. Kürbiskeimlinge A: 12,977 Grm. Trockensubst. wurden zuerst in der Kälte, dann in der Wärme mit 85 proc. Weingeist extrahirt. Der Extrakt wurde unter Zusatz von etwas Kalkmilch eingedampft, der Rückstand in Wasser aufgenommen, die Lösung unter Zusatz von etwas Bleiessig anf $110 \mathrm{Ccm}$. gebracht, dann filtrirt. $50 \mathrm{Ccm}$. des Filtrats gaben beim Kochen mit Eisen- 


\section{Schulze: Zur Kenntniss der stickstoffhaltigen}

Gehalt der Keimpflanzentrockensubst. an Kaliumnitrat:

Keimlinge A, ca. 3 Wochen alt $0,614 \%$ " B, "2 " " 21,564 ,

In den lufttrocknen Axenorganen einer dritten Vegetation (C) wurden nach der gleichen Methode 0,344\% Kaliumnitrat gefunden.

In einigen anderen in gleicher Weise gezogenen Vegetationen solcher Keimlinge waren aber nur Spuren von Nitraten nachzuweisen.

Wir haben schliesslich noch Kürbiskeimlinge untersucht, welche in zuvor ausgeglühtem und sodann mit Wasser sorgfältig ausgewaschenem Sand gezogen und während des Wachsthums nur mit destillirtem Wasser begossen worden waren. Sie erhielten nach dem Ergebniss einer nach Schlö. sing's Methode ausgeführten Bestimmung in der Trockensubstanz $0,436 \%$ Salpetersäure $=0,70 \%$ Kaliumnitrat.

Bei Erörterung der Frage nach dem Ursprunge der in den Keimlingen aufgefundenen Nitrate ist zunächst zu bemerken, dass die ungekeimten Kürbissamen nachweisbare Mengen solcher Salze nicht enthielten. Dass die Nitrate aus dem Sande stammten, in welchem die Keimlinge erwachsen waren, ist nicht anzunehmen; sollten in demselben ursprünglich solche Salze in geringer Menge sich vorgefunden haben, so müssten sie doch zweifellos durch das Aus-

chlorür und Salzsäure nach Schlösing's Methode 10,0 Ccm. NO bei $17^{\circ}$ und $725 \mathrm{Mm}$. Barometerstand $=0,02127 \mathrm{Grm} . \mathrm{N}_{2} \mathrm{O}_{5}$.

K ürbiskeimlinge B: 7,0958 Grm. Trockensubst. wurden mit 90 proc. Weingeist in der Wärme extrahirt, der Extract wie oben behandelt, dann ganz für die Bestimmung verwendet, Erhalten $27,8 \mathrm{Ccm}$. $\mathrm{NO}$ bei $18,5^{\circ}$ und $730 \mathrm{Mm}$. Barometerstand $=0,0592697 \mathrm{Grm} . \mathrm{N}_{2} \mathrm{O}_{5}$.

Axenorgane der Kürbiskeimlinge C: $10 \mathrm{Grm}$. Trockensubstanz wurden mit 90 proc. Alkohol in der Wärme extrahirt, Extract wie oben behandeit, ganz zur Bestimmung verwendet. Erhalten 8,6 Ccm. NO bei $15,5^{\circ}$ und $723 \mathrm{Mm}$. Barometerstand $=0,018369 \mathrm{Grm} . \mathrm{N}_{2} \mathrm{O}_{5}$.

K ürbiskeimling e D: 9,980 Grm. Trockensubstanz, mit Weingeist extrahirt, Extract wie oben behandelt. Erhalten $15,4 \mathrm{Ccm}$. NO, redncirt auf $0^{\circ}$ und $760 \mathrm{Mm}$. Bar. $=0,03722 \mathrm{Grm} . \mathrm{N}_{2} \mathrm{O}_{5}$. 
waschen entfernt worden sein. $^{1}$ ) Das Wasser, mit welchem die Keimlinge begossen wurden, war, wie früher schon erwähnt worden ist, destillirtes Wasser. Es ist also nicht ersichtlich, wie die Nitrate von aussen in die Keimlinge hineingekommen sein könnten; man muss daher wohl annehmen, dass sie sich in den letzteren gebildet haben.

Bekanntlich haben Berthelot und André) vor Kurzem auf Grund ausgedehnter Untersuchungen den höheren Pffanzen die Fähigkeit zugesprochen, in ihrem Organismus Nitrate zu bilden, nachdem solches für niedere Organismen früher schon durch Schlösing und Müntz nachgewiesen worden war.

Auffallend sind die grossen Schwankungen, welche im Nitratgehalt der von uns untersuchten Kürbiskeimlinge sich zeigten. Welche Umstände von Einfluss darauf waren, lässt sich aus unseren Untersuchungen nicht ableiten.

Den im Vorigen über den Stoffgehalt der Kürbiskeimlinge gemachten Mittheilungen füge ich noch einige.Angaben über die Quantität an, in welcher einige der organischen Bestandtheile sich vorfanden.

Auf den Gehalt der Keimlinge an Tyrosin und an Leucin können wir nur aus der Ausbeute schliessen, welche bei Darstellung der genannten Amidosäuren sich ergab. J. Barbieri und ich erhielten pro 100 Thle. der Keimpflanzentrockensubstanz 0,2-0,25 Gr. Tyrosin. Die Ausbeute an Leucin war keine grössere; bei Verarbeitung der Axen-

1) Den für die späteren Versuche verwendeten Flusssand untersuchten wir vor der Verwendung auf Nitrate. Wir extrahirten eine Portion mit destillirtem Wasser, verdampften den Extract bis fast zur Troekne, setzten dann concentrirte Schwefelsäure und etwas Bucin hinzu. Es entstand eine röthliche Färbung; demuach können minimale Nitratmengen vorhanden gewesen sein. Dieselben sind aber zweifellos durch das nachfolgende Auswaschen des Sandes entfernt worden.

2) Chemisches Centralbl. 1884, S. 939, nach Compt. rend. 99, $683-688$. 


\section{Schulze: Zur Kenntniss der stickstoffhaltigen}

organe erhielten wir aus $1 \mathrm{Kgr}$. Trockensubstanz kaum $2 \mathrm{Gr}$. Leucin. Da ein Theil des letzteren ohne Zweifel in der dickflüssigen Mutterlauge zurückblieb, so ist anzunehmen, dass in Wirklichkeit eine jene Zahl übersteigende Quantität vorhanden war.

Die Glutamin - und Asparaginmenge, welche in den Keimlingen in maximo vorhanden gewesen sein kann, lässt sich aus der beim Kochen mit Salzsärure in den Extracten sich bildenden Ammoniakquantität berechnen; letztere ergibt sich, wenn man von der Ammoniakmenge, welche nach dem Kochen mit Salzsäure im Ganzen vorgefunden wird, das ursprünglich schon vorhandene Ammoniak subtrahirt. Die Bestimmung der letzteren bietet wegen der leichten Zersetzbarkeit des Glutamins einige Schwierigkeiten dar. Auf Grund der von E. Bosshard ${ }^{1}$ ) ausgeführten Versuche erschien es uns als das zweckmässigste, das Ammoniak durch Phosphorwolframsäure auszufällen und aus dem Niederschlag durch Destillation mit Wasser und gebrannter Magnesia zu gewinnen. Das Glutamin wird durch Phosphorwolframsäure nicht gefällt, geht also ins Filtrat über.

Ueber die Details der Versuchsausführung ist Folgendes zu bemerken: Die frischen Keimlinge wurden durch Waschen mit Wasser vom anhängenden Sand befreit und sodann sorg. fältig mit Fliesspapier abgetrocknet. Eine abgewogene Durchschnittsprobe dieser Keimlinge verwendeten wir zur Bestimmung des Trockengehalts ${ }^{2}$ ), eine andere zur Darstellung eines wässrigen Extracts. Die letztere Probe wurde unter Zusatz von Sand zerrieben, der Brei auf ein Zeugfilter gebracht, mit Wasser gewaschen, abgepresst, die ablaufende trübe Flüssigkeit bis zum Coaguliren der Eiweissstoffe erhitzt, dann filtrirt; das Filtrat versetzten wir mit Bleizuckerlösung unter Vermeidung eines Ueberschusses (in einem Falle zuvor mit etwas Gerbsäure), füllten die Flüssigkeit mit dem

1) Zeitschr. analyt. Chem. 22, 329.

2) Da die frischen Keimlinge sehr wasserreich sind (ihr Trockengehalt schwankte von $5-8 \%$ ) und durch Verdunstung fortwährend Wasser abgeben, so ist eine ganz genaue Bestimmung des Trockengehaltes kaum ausführbar. 
Niederschlag auf ein bestimmtes Volumen auf und filtrirten. In einem Theile des Filtrats bestimmten wir das Ammoniak unter Befolgung der von E. Bosshard gegebenen Vorschriften ${ }^{1}$ ); einen anderen Theil kochten wir $2^{1} / 2$ Stunden lang mit Salzsäure und bestimmten sodann den Ammoniakgehalt durch Destillation mit Magnesia; das ausgetriebene Ammoniak wurde in verdünnter Schwefelsäure aufgefangen, letztere mit Barytwasser zurücktitrirt. Wir fanden so folgende Ammoniakmengen (angegeben in Procenten der Keimpflanzentrockensubstanz ${ }^{2}$ ):

1) A. a. 0 .

2) Analytische Belege. A. 12-13tägige Keimlinge: Extract aus 148,35 Grm. frischen Keimlingen (mit 11,364 Grm. Trockensubstanz) wurde auf $100 \mathrm{Ccm}$. gebracht.

1) Bestimmung des vor dem Kochen mit Salzsäure vorhandenen Ammoniaks: $100 \mathrm{Ccm}$. des obigen Extractes gaben:

a. 0,0038978 Grm. $\mathrm{N}$ in Ammoniakform (=1,0 Ccm. Barytlauge $\alpha$ ).

b. $0,003703 \quad ", \quad$ " $"=0,95, \quad, \alpha)$.

2) Bestimmung des nach dem Kochen mit Salzsäure vorhandenen Ammoniaks: $150 \mathrm{Ccm}$. des obigen Extractes wurden mit $10 \mathrm{Ccm}$. concentrirter Salzsäure 21/2 Stunden lang gekocht, dann annähernd mit Natronlange neutralisirt und auf $200 \mathrm{Ccm}$. gebracht. Je $100 \mathrm{Ccm}$ davon gaben:

a. 0,010134 Grm. $\mathrm{N}$ in Ammoniakform ( $=2,6 \mathrm{Ccm}$. Barytlauge $\alpha$ ).

b. $0,0099395, ", \quad$ " $"=2,55, \quad, \quad \alpha)$.

B. 18-19tägige Keimlinge: Extract aus 108,8 Grm. frischen Keimlingen mit 4,872 Grm. Trockensubst. auf $350 \mathrm{Ccm}$. gebracht.

1) Bestimmung des vor dem Kochen mit Salzsäure vorhandenen Ammoniaks: $100 \mathrm{Ccm}$. des Extractes gaben 0,005086 Grm. $\mathrm{N}$ in Ammoniakform $(=1,3 \mathrm{Ccm}$. Barytlange $\beta$ ).

2) Bestimmung des nach dem Kochen mit Salzsäure vorhandenen Ammoniaks: $150 \mathrm{Ccm}$. des obigen Extractes wurden mit Salzsäure gekocht, dann auf $200 \mathrm{Ccm}$. gebracht. Je $100 \mathrm{Ccm}$. davon gaben:

a. 0,009389 Grm. $\mathbf{N}$ in Ammoniakform $(=2,4 \mathrm{Ccm}$. Barytlauge $\beta$ ).

b. $0,009389 \quad " n \quad " \quad(=2,4 \quad " \quad, \quad \alpha)$.

C. 20-21tägige Keimlinge: Extract aus $340 \mathrm{Grm}$, frischen Keimlingen (mit 21,68 Grm. Trockensubst.) auf $620 \mathrm{Ccm}$. gebracht.

1) Bestimmung des vor dem Kochen mit Salzsäure vorhandenen Ammoniaks: $100 \mathrm{Ccm}$. des Extractes gaben 0,0077956 Grm. $\mathbf{N}$ in Ammoniak form $(=2,0 \mathrm{Ccm}$. Barytlauge $\omega)$.

2) Bestimmung des nach dem Kochen mit Salzsäure vorhandenen 


\begin{tabular}{|c|c|c|c|c|c|c|}
\hline & & & & a. Vor & b. Nach & c. Differenz. \\
\hline & & & & Kochen & it Salzsäur & \\
\hline Kürbiskeimlinge & $12-13$ & Tage & alt & $0,204 \%$ & $0,714 \%$ & $0,510 \% \mathrm{NH}_{3}$ \\
\hline$"$ & $18-19$ & " & $"$ & 0,443, & 1,091, & $0,648, "$, \\
\hline$"$ & $20-21$ & $n$ & 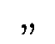 & 0,271 & 1,012, & 0,741 \\
\hline
\end{tabular}

Unter der Voraussetzung, dass die in der dritten Columne aufgeführten Ammoniakmengen ausschliesslich durch Zersetzung von Glutamin entstanden sind, würde sich für die untersuchten Keimlinge folgender Glutamingehalt berechnen:

Kürbiskeimlinge $12-13$ Tage alt $4,38 \%$ Glutamin $(=0,840 \%$ N)

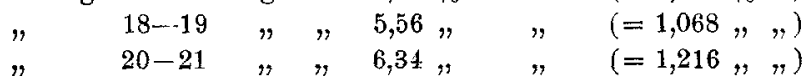

Dass die der vorstehenden Rechnung zu Grunde liegende Voraussetzung keine ganz richtige ist, geht schon daraus hervor, dass die Kürbiskeimlinge nach unseren Untersuchungen auch ein wenig Asparagin enthalten. Der durch diesen Umstand bedingte Fehler ist jedoch nur ein geringfügiger (der in der beschriebenen Weise berechnete Glutamingehalt schliesst das Asparagin mit ein). $\mathrm{Ob}$ aber in den für die Bestimmung verwendeten Extracten neben Glutamin und Asparagin nicht noch andere, durch Salzsäure unter Ammoniakbildung zersetzbare Substanzen vorhanden waren, lässt sich zur Zeit nicht entscheiden ${ }^{1}$ ).

Ammoniaks: $200 \mathrm{Ccm}$. des obigen Extractes wurden mit Salzsäure gekocht, dann auf $250 \mathrm{Ccm}$. gebracht. Je $100 \mathrm{Ccm}$. davon gaben:

a, $0,023192 \mathrm{Grm}$. $\mathbf{N}$ in Ámmoniakform $(=5,95 \mathrm{Ccm}$. Barytlauge $\alpha)$. b. $0,023387 \quad, \quad, \quad(=6,0 \quad, \quad$ " $"$ a).

Titre der Barytlauge $\alpha: 1 \mathrm{Ccm} .=0,0038978 \mathrm{Grm} . \mathrm{N}$. $" \quad, \quad \beta: 1 \quad,=0,003912 \quad, \quad$,

1) In Betreff dieser Frage sind wir also durch die neueren Untersuchungen nicht weiter gekommen; schon in der früheren Abhandlung mussten wir es als fraglich bezeichnen, ob in den Kürbiskeimlingen neben Glutamin und Asparagin nicht noch andere, durch Salzsäure zersetzbare Stickstoffverbindungen vorhanden sind. Die Glutaminmenge, welche aus den Keimlingen zux Abscheidung gebracht werden kann, bleibt ohne Zweifel hinter dem in der obigen Weise berechneten Glutamingehalt weit zurück; genaue Zahlen für die Glutaminausbeute können wir zur Zeit nicht geben, weil weder Trockengehaltsbestimmungen der für die Glutamindarstellung verwendeten Keimlinge, noch 
Die Stickstoffmenge, welche nach möglichst vollständiger Entfernung der Eiweissstoffe in den Extracten sich noch vorfand, betrug bei 15 tägigen Keimlingen nach einer früher ausgeführten Bestimmung $\left.{ }^{1}\right) 3,19 \%$, bei $20-21$ täg. Keimlingen $3,48 \%$, bezogen auf die Keimpfianzentrockensubstanz. Auf Glutamin und Asparagin fällt nur etwa der dritte Theil dieser Stickstoffmenge; ungefähr $2 \% \mathrm{~N}$ müssen in Form anderer Verbindungen vorhanden gewesen sein. Nun sind ja in den Extracten zahlreiche andere stickstoffhaltige Körper von uns nachgewiesen worden, nämlich Amidosäuren, Xanthinkörper, Ammoniaksalze, Nitrate etc.; da aber keiner dieser Bestandtheile in sehr grosser Menge vorhanden war, so ist es immerhin fraglich, ob durch dieselben jene Stickstoffmenge vollständig gedeckt wird, oder ob nicht vielleicht die Extracte neben jenen Körpern noch andere, bisher nicht aufgefundene stickstoffhaltige Stoffe enthielten.

\section{Rückblick auf die Resultate.}

In den etiolirten Kürbiskeimlingen finden sich nach unseren Untersuchungen Glutamin, Asparagin, Leucin, Tyrosin, Vernin, Xanthinkörper, Ammoniaksalze und Nitrate vor. Die meisten dieser Stoffe sind vermuthlich sowohl in den Cotyledonen wie in den Axenorganen der Keimlinge enthalten; für einige derselben haben wir jedoch bis jetzt nur das Vorkommen am einen oder anderen

Bestimmungen des Mengenverhältnisses zwischen Cotyledonen und Axenorganen gemacht worden sind. Uebrigens haben Zahlenangaben für die Glutaminausbeute auch deshalb wenig Werth, weil anzunehmen $\mathbf{i}_{\text {st }}$, dass ein Körper, welcher durch Beimengungen bisweilen ganz am Auskrystallisiren gehindert wird (wie es für das Glutamin von uns beobachtet wurde), stets nur partiell aus den Extracten auskrystallisirt.

1) Das Ergebniss dieser Bestimmung ist in der früheren $\mathrm{Ab}$ handlung (S. 409) schon mitgetheilt worden. Damals haben wir den Extract durch Behandlung mit Bleioxydhydrat (nach der Methode von F. Hofmeister) von dem beim Erhitzen etwa noch in Lösung gebliebenen Rest der Eiweisskörper zu befreien gesucht, bei Ausführung der späteren Bestimmung dagegen durch Kochen mit einer Lösung von essigsaurem Eisenoxyd. 


\section{Schulze: Zur Kenntniss der stickstoffhaltigen}

Ort nachweisen können. So z. B. fanden wir Asparagin und Vernin nur in den Cotyledonen; Glutamin haben wir bis jetzt nur aus den Axenorganen erhalten; doch ist es sehr wohl möglich, dass dieser Körper auch in den Cotyledonen vorhanden war, aber durch Beimengungen an der Ausscheidung aus den Extracten gehindert wurde. Tyrosin und Xanthinkörper wurden sowohl in den Cotyledonen wie in den Axenorganen nachgewiesen. Was die Ammoniaksalze und die Nitrate betrifft, so haben wir über den Ort ihres Vorkommens keine Untersuchungen angestellt.

Es scheint, dass der Gehalt der Kürbiskeimlinge an den oben genannten Produkten gewissen Schwankungen unterliegt. Einige Vegetationen jener Keimlinge enthielten so viel Asparagin, dass man annehmen muss, es sei in denselben das Glatamin durch Asparagin ersetzt gewesen. In einigen Fällen fanden wir in den Cotyledonen eine nicht ganz unbeträchtliche Menge von Vernin, in anderen nur äusserst wenig davon vor.

Von den oben aufgeführten Substanzen können vier mit grosser Wahrscheinlichkeit für Produkte des mit dem Keimungsvorgang verbundenen Zerfalls von Eiweissmolekülen erklärt werden, nämlich das Glutamin, das Asparagin, das Leucin und das Tyrosin; denn die beiden letzteren Körper und die den ersteren entsprechenden Amidosäuren werden ja bei der Spaltung der Fiweisskörper ausserhalb des Organismus stets erhalten. Die Xanthinkörper können auf Grund der von A. Kossel ${ }^{1}$ ) ausgeführten Untersuchungen für Zersetzungsprodukte der Nucleïne angesehen werden. Ueber den Ursprung der in den Keimlingen vorgefundenen Ammoniaksalze und Nitrate lässt sich zur Zeit etwas Bestimmtes nicht aussagen.

Es ist von Interesse, die Kürbiskeimlinge in Bezug auf ihren Gehalt an Produkten der regressiven Stoffmetamorphose mit den von uns gleichfalls möglichst eingehend untersuchten etiolirten Keimlingen der gelben Lupine ${ }^{2}$ ) zu

1) Zeitschr. f. physiolog. Chem. 5, 267 u. 6, 422.

2) Vergl dies. Journ. [2] 27, 337-362. 
vergleichen. In letzteren finden sich als Bestandtheile, welche mit grosser Wahrscheinlichkeit als Produkte der Eiweisszersetzung zu erklären sind, Asparagin, Phenylamidopropionsäure und Amidovaleriansäure ${ }^{1}$ ) vor; Leucin und Tyrosin sind wahrscheinlich in geringer Menge gleichfalls vorhanden, aber bis jetzt nicht mit aller Sicherheit nachgewiesen. Die Anwesenheit von Xanthinkörpern ist constatirt, A m moniak salze scheinen bisweilen nur in äusserst geringer, bisweilen in etwas grösserer Quantität vorhanden zu sein.

Zwischen den Kürbis- und den Lupinenkeimlingen zeigt sich also mit Bezug auf den Gehalt an Körpern, welche man als Produlte des Eiweisszerfalls betrachten darf, eine bemerkenswerthe Differenz. Allerdings ist es sehr wohl möglich, dass Amidovaleriansäure und Phenylamidopropionsäure, welche aus den Keimlingen der gelben Lupine, nicht aus denen des Kürbis abgeschieden werden konnten, auch in den letzteren in geringer Menge sich vorfinden ${ }^{2}$ ); dass

1) Das Asparagin kann sogar mit völliger Bestimmtheit als Eiweisszersetzungsprodukt betrachtet werden; denn es findet sich in so grosser Menge in den Lupinenkeimlingen vor, dass die neben Eiweissstoffen in den ungekeimten Lupinensamen noch vorhandenen Stickstoffverbindungen das Material zu seiner Bildung nicht geliefert haben köonen. - Es sei hier erwihnt, dass wir auch bei einer späteren Untersuchung von Lupinenkeimlingen in denselben neben Aspa ragin und Phenylamidopropionsäure wieder Amidovaleriansäure vorfanden. Die letztere wurde durch Stickstoffbestimmungen nach Kjeldahl's Methode jdentificirt (ausgeführt von E. Bosshard), welche folgende Resultate gaben:

$$
\begin{aligned}
& \text { Burechnet } \\
& \text { für } \mathrm{C}_{\overline{1}} \mathrm{H}_{11} \mathrm{NO}_{2} \text {. } \\
& \text { N } \quad 11,96 \% \\
& 1 . \\
& 11,89 \% \\
& \text { Gefunden. } \\
& 2 . \\
& 3 . \\
& 11,65 \% \quad 11,72 \%
\end{aligned}
$$

Für diese Bestimmungen wurden zwei Präparate verwendet; es ist möglich, dass das eine derselben nicht ganz frei von Leucin war.

2) Es liegt auf der Hand, dass Bestandtheile, welche nur in sehr geringer Menge vorkommen, aus den Extracten oft nicht zur Abscheilung gebracht werden können. Für das Vorhandensein einer geringen Menge von Phenylamidopropionsäure in den Kürbiskeimlingen spricht ja übrigens die früher (auf S. 446) von uns mitgetheilte Beobachtung. 
andererseits auch die Iupinenkeimlinge eine sehr geringe Quantität von Glutamin enthalten - dass demnach in quali tativer Hinsicht im Gehalt der genannten Keimlinge an Eiweisszersetzungsprodukten eine Verschiedenheit eigentlich nicht obwaltet. Dagegen kann es keinem Zweifel unterliegen, dass in Bezug auf die Quantität der einzelnen Produkte grosse Unterschiede zwischen den genannten Keimlingen vorhanden sind.

Eine Erklärung einer solchen Erscheinung hat der Vert. schon früher zu geben versucht. ${ }^{1}$ ) Es ist nicht unwahrscheinlich, dass wir bei der Untersuchung der Keimpflanzen die Eiweisszersetzungsprodukte nicht mehr in demjenigen Mengenverhältniss vorfinden, in welchem sie beim Zerfall der Eiweissmoleküle ursprünglich entstanden sind. Denn in den wachsenden Keimpflanzen geht nicht nur Eiweisszerfall, sondern auch Regenerirung von Eiweissstoffen auf Kosten der Zerfallsprodukte vor. Es ist nun möglich, dass für letzteren Zweck die einzelnen Spaltungsprodukte nicht stets in gleichem Maasse verbraucht werden; ist dies der Fall, 50 werden wir in den Keimlingen in grösserer Menge nur diejenigen Produkte vorfinden, welche langsam wieder zum Verbrauch gelangen.

Hält man eine solche Erklärung ${ }^{2}$ ) nicht für zulässig, so würde man annehmen müssen, dass die Quantitiit, in welcher beim Zerfall eines Eiweissstoffes die einzelnen Amidosäuren sich bilden, überhaupt keine constante ist, sondern je nach den Umständen wechselt; den beim Studium der Zersetzung der Eiweisskörper bis jetzt gemachten Beobachtungen können aber wohl kaum Stützen für die letztere Annahme entnommen werden. ${ }^{3}$ )

Zürich, agric.-chem. Laborat. d. Polytechn., im Juli 1885.

1) Landwirthschaftl. Jahrb, herausgeg. v. H. Thiel, 9, $716 \mathrm{ff}$.

2) In Betreff der eiuzelnen Thatsachen und Erwägungen, welche dieser Erklärungsweise als Stützen dienen können, sei auf die oben citirte Abhandlung verwiesen.

3) Auch würde eine solche Annahme im Widerspruch mit der vielfach geäusserten Anscbauung stehen, dass Amidosiurenreste präformirt im Eiweissmolekül sich vorfinden. 